\title{
Delayed matching in the pigeon: Interference produced by the prior delayed matching trial
}

\author{
DAVID E. HOGAN \\ Northern Kentucky University, Highland Heights, Kentucky 41076 \\ and \\ CHARLES A. EDWARDS and THOMAS R. ZENTALL \\ University of Kentucky, Lexington, Kentucky 40506
}

\begin{abstract}
Pigeons' delayed matching performance on Trial $n$ was examined as a function of whether the correct and incorrect comparison stimuli from Trial $n-1$ were maintained in the same role on Trial $\mathbf{n}$ (positive transitions), were reversed in role on Trial $\mathbf{n}$ (negative transitions), or were absent on Trial $\mathbf{n}$ (neutral transitions). Relative to neutral transitions, positive transitions did not significantly facilitate performance. Negative transitions, however, produced significant proactive interference on Trial $n$, and the magnitude of proactive interference was greater when the Trial $\mathrm{n}$ retention interval was $1 \mathrm{sec}$ than when it was $0 \mathrm{sec}$. As the intertrial interval increased from 2 to $10 \mathrm{sec}$, the amount of interference dissipated. The results suggest that a prior delayed matching trial can serve as a significant source of forgetting but not a significant source of facilitation on an immediately following delayed matching trial.
\end{abstract}

The delayed matching-to-sample task has become a popular tool for evaluating short-term memory loss in pigeons (e.g., Roberts \& Grant, 1976; Zentall \& Hogan, 1977). In the delayed matching task, the subject is presented with a sample stimulus to which an observing response is required. Following a brief delay interval in the absence of the sample, two comparison stimuli are presented, one that matches the sample and another that differs from the sample. A response to the matching comparison stimulus is defined as correct.

It is typically observed that pigeons' delayed matching performance declines rather rapidly as the retention interval between the sample and comparison stimuli is lengthened. Although the decline in matching accuracy has been attributed to a spontaneous weakening of the memory trace of the sample stimulus (Roberts, 1972), recent data indicate that forgetting may be attributed to a proactive influence of stimuli presented before the sample stimulus (proactive interference) (Grant, 1975; Grant \& Roberts, 1973; Zentall \& Hogan, 1977).

In a recent study of proactive interference (Zentall \& Hogan, 1977), pigeons' delayed matching performance was compared with a single proactive stimulus

The research was supported by Grant $\mathrm{MH} 24092$ from the National Institute of Mental Health to T. R. Zentall. Preparation of the manuscript was supported by a Northern Kentucky University Faculty Summer Fellowship awarded to D. E. Hogan. We thank Tammy Rayburn for her assistance with the manuscript. Reprint requests should be sent to David E. Hogan, Department of Psychology, Northern Kentucky University, Highland Heights, Kentucky 41076. presented before the sample and with a control condition with no interfering proactive stimulus. Results indicated that the amount of interference produced by a proactive stimulus depended primarily on (1) whether or not on previous trials the proactive stimulus had been associated with a response to the incorrect comparison stimulus on the matching trial, and (2) given its association with the incorrect comparison stimulus, whether the proactive stimulus occurred in close temporal proximity to the sample. When the proactive stimulus was presented immediately before the sample, an equivalent amount of interference resulted after a 0 -sec and a 1 -sec samplecomparison retention interval. However, when the proactive stimulus was separated from the sample by a neutral stimulus, relatively more interference was produced at the $1-\mathrm{sec}$ retention interval than at the 0 -sec retention interval.

Zentall and Hogan proposed that a temporal discrimination hypothesis (D'Amato, 1973) could account for their results. According to this hypothesis, delayed matching-to-sample involves selecting that comparison stimulus which was presented most recently as the sample. The decline in matching accuracy across increasing retention intervals is a result of the pigeon's failure to discriminate whether a proactive stimulus or the sample stimulus was seen last. Furthermore, temporal discriminability is affected not only by the absolute length of the retention interval, but also by the time interval between the proactive stimulus and the sample. When a proactive stimulus is presented immediately before the sample, it is difficult for the pigeon to remember if the sample 
had been presented last, even at short retention intervals. However, when a proactive stimulus is separated in time from the sample, the pigeon can easily remember that the sample was presented last if the retention interval is short (e.g., zero delay), but performance suffers (relative to control performance) if the retention interval is long.

The intratrial proactive stimulus procedure provides a simple source of proactive interference, but it does not necessarily reflect the intertrial proactive effects that may be present in a standard matchingto-sample task. In the present experiment, pigeons' delayed matching performance was examined when the immediately preceding delayed matching trial served as the proactive event. Delayed matching performance was examined on Trial $\mathrm{n}$ as a function of whether it was preceded by a positive, neutral, or negative transition trial $(n-1)$. On positive transition trials, the sample and incorrect comparison stimuli of Trial $n-1$ were repeated in the same role on Trial $n$. For example, if Trial $n-1$ involved a red correct comparison stimulus, a red sample, and a green incorrect comparison stimulus (RRG), then Trial n was also RRG. On neutral transition trials, the sample and incorrect comparison stimuli of Trial $n-1$ were both absent on Trial $n$ (e.g., if Trial $n-1$ was $R R G$, then Trial $n$ was either BBY or YYB). On negative transition trials, the sample and incorrect comparison stimuli of Trial $n-1$ were reversed in role on Trial $n$ (e.g., if Trial $n-1$ was RRG, then Trial $n$ was GGR). The retention interval, which varied within sessions, was either 0 or $1 \mathrm{sec}$. The intertrial interval (ITI), which varied between sessions, was 2,5 , or $10 \mathrm{sec}$.

In general, temporal discrimination theory would predict that the amount of interference produced by a negative transition trial should be larger when the ITI was short and dissipate as the ITI was lengthened. Furthermore, one would expect interference to be greater when the retention interval was long, since the difficulty in discriminating the sample of Trial $n$ from that of Trial $n-1$ should increase as the retention interval was lengthened. Since similar predictions would be made by trace-decay theory, the inclusion of positive transition trials provided a more direct test of temporal discrimination theory. According to temporal discrimination theory, there should be no proactive facilitation from a positive transition trial, relative to neutral transition trials, since a temporal discrimination is not necessary unless the prior trial involves the incorrect comparison stimulus and thus produces competing memories at the time of the retention test.

\section{METHOD}

\section{Subjects}

Eight loft-reared pigeons, maintained at $80 \%$ of their freefeeding weights, served as subjects. All pigeons had had prior experience matching red, green, yellow, and blue hues in a simultaneous matching procedure (see Zentall \& Hogan, 1978, for the pretraining and training regimen).

\section{Apparatus}

The apparatus used was a sound-attenuated chamber, $36.8 \mathrm{~cm}$ high, $30.8 \mathrm{~cm}$ wide, and $34.3 \mathrm{~cm}$ deep. Three pecking keys, $2.7 \mathrm{~cm}$ wide and $2.5 \mathrm{~cm}$ high, were mounted on the front wall. Adjacent keys were separated by a $.5-\mathrm{cm}$ barrier to discourage pigeons from pecking adjacent keys simultaneously. Centered behind each pecking key was an in-line projector capable of presenting four hues: red, green, yellow, and blue (Kodak Wratten filters, 26, 60,9 , and $38 \mathrm{~A}$, respectively). Control equipment was located in an adjoining room. White noise and a blower fan served to mask extraneous noise.

\section{Procedure}

Preliminary training. All pigeons were exposed to delayed matching trials in which the sample stimulus was red or green and the comparison stimuli were red and green and to trials in which the sample stimulus was blue or yellow and the comparisons were blue and yellow. The onset of a trial was signaled by the illumination of a dim houselight, and a small circular spot $(.5 \mathrm{~cm}$ diam) on the center key, which served as a "ready signal." A single peck to the ready signal immediately produced one of the four sample hues. Five pecks to the sample stimulus turned off the sample and initiated either a 0 or a $1.0-\mathrm{sec}$ retention interval, followed by presentation of the comparison stimuli. A single peck to the matching comparison stimulus was followed by $2-\mathrm{sec}$ access to Purina Pigeon Grains and a 5-sec ITI. During the ITI, the houselight and response keys were darkened. A peck to the comparison stimulus which was different from the sample hue was followed only by the ITI.

Daily sessions consisted of 96 trials. The sample hue and position of the correct comparison stimulus were counterbalanced within each session. The retention intervals $(0$ and $1 \mathrm{sec})$ were counterbalanced across sample hue and correct comparison position. The order of training trials was determined randomly, with the constraint that a particular comparison position or comparison hue not be permitted to be correct on more than three consecutive trials. Preliminary training was continued for 10 sessions.

Phase 1. The procedure during Phase 1 was the same as during preliminary training, except that the sample stimulus duration was fixed at $1 \mathrm{sec}$, independent of sample pecks. A peck to the ready signal was still required.

Each trial served as both a proactive trial (Trial $n-1)$ and as a test trial (Trial $n$ ). Each session consisted of 97 trials. The first trial of each session served as a "dummy trial" which did not enter into the calculation of test trial performance.

Three types of trial transitions were presented within each session: (1) In positive transitions ( 32 trials), the sample and incorrect hues of Trial $n-1$ were repeated in the same role on Trial $n$ (e.g., if Trial $n-1$ involved a red sample and a choice between a red and a green comparison key, then Trial $\mathrm{n}$ was the same). The position of the correct comparison on Trial $n$ was either the same as that of Trial $n-1$ (16 trials) or different from that of Trial $n-1$ (16 trials). (2) In neutral transitions (32 trials), the sample and incorrect comparison hues of Trial $n-1$ were both absent on Trial $n$ (e.g., if Trial $n-1$ involved a red sample and a choice between $a$ red and a green comparison key, then Trial $n$ involved either a blue or yellow sample and a choice between a blue and a yellow comparison key). Again, the position of the correct comparison stimulus on Trial $n$ was either the same as that of Trial $n-1$ (16 trials) or different from that of Trial $n-1$ (16 trials). (3) In negative transitions ( 32 trials), the sample and incorrect comparison hues of Trial $n-1$ were reversed in role on Trial $n$ (e.g., if Trial $n-1$ involved a red sample and a choice between a red and a green comparison $k e y$, then Trial $n$ involved a green sample and a choice between a red and a green comparison key). Again, the correct comparison position of Trial $n$ was either the same as that of Trial $n-1$ (16 trials) or different from that of Trial $n-1$ (16 trials). 
Transitions were counterbalanced for sample hue, retention interval, and correct comparison position. Four different trial orders (randomly selected) were used during Phase 1 . The ITI $(2,5$, or $10 \mathrm{sec}$ ) was varied across sessions in an ABC, CBA order. Phase 1 consisted of 24 sessions.

Phase 2. Phase 2 was identical to Phase 1 , except that the sample stimulus duration was reduced from 1.0 to $.5 \mathrm{sec}$. Phase 2 consisted of 48 sessions.

\section{RESULTS}

Delayed matching performance during the last six sessions of preliminary training averaged $87 \%$ correct on 0 -sec delay trials and $76 \%$ correct on $1-\mathrm{sec}$ delay trials. The data were subjected to a repeated measures analysis of variance involving delay and sessions as factors. For this, and all subsequent analyses, a rejection region of $p<.05$ was adopted.

The analysis indicated a significant effect of delay $[F(1,7)=15.29]$ but nonsignificant effects of sessions $[F(5,35)=1.32]$ and delay $\times$ sessions interaction $(F<1)$. Thus, there was stable performance at each retention interval prior to Phase 1 .

\section{Phase 1}

Mean performance for each transition (pooled over subjects and sessions) is plotted in Figure 1 as a function of the retention interval for each ITI duration. When the proactive trial was separated from the test trial by a 2 -sec ITI, there were two noteworthy outcomes. First, there was no apparent difference between performance on positive and neutral transition trials at either the 0 - or the $1-\mathrm{sec}$ retention intervals. Second, relative to performance on neutral transition trials, performance was poorer on negative transition trials and the difference increased as the retention interval increased.

Performance functions for the 5-sec ITI condition were quite comparable to those for the 2 -sec ITI con-

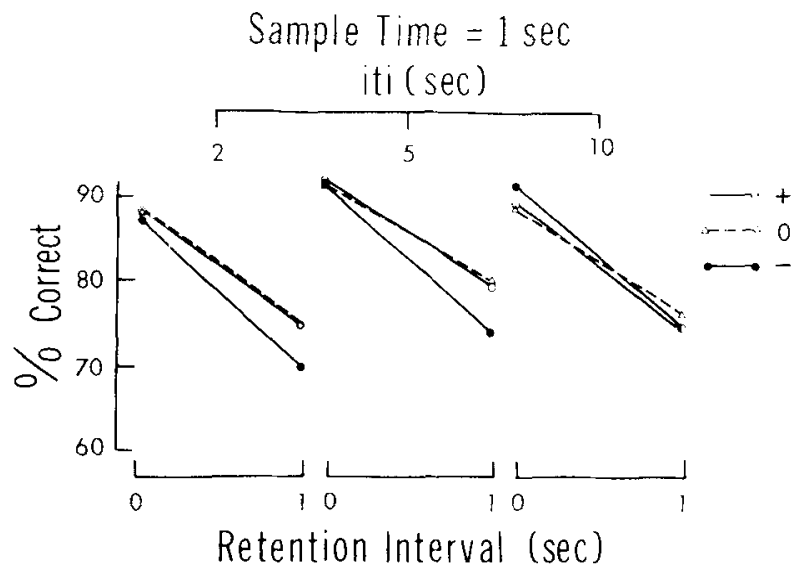

Figure 1. Phase 1: Performance on positive $(+)$, neutral (0), and negative ( - ) transition trials as a function of the retention interval and ITI condition when the sample duration was 1 sec. dition. There was little difference between performance on positive and neutral transition trials at either retention interval, and, again, performance on negative transition trials diverged from performance on neutral transition trials as the retention interval increased. In the 10-sec ITI condition, there was virtually no difference among transitions at either retention interval.

An analysis of main effects indicated significant effects of delay $[F(1,7)=31.3]$ and ITI $[F(2,14)=$ $4.97]$, but not of transition $[F(2,14)=1.90]$. Further analyses indicated that performance at the 2-sec ITI was significantly inferior to the combined performance at the 5- and 10 -sec ITI $[F(1,7)=6.30]$, whereas performance at the 10 -sec ITI did not differ significantly from performance at the 5-sec ITI $[F(1,7)=$ 2.46].

The transition $\times$ delay interaction was significant $[F(2,14)=4.95]$, and further analyses indicated that performance on negative transitions diverged significantly from the combined performance on positive and neutral transitions as the retention interval increased $[F(1,7)=24.01]$. However, the magnitude of the difference between positive and neutral transitions did not change as the retention interval increased $(F<1)$.

Although the difference between negative and neutral transition performance at the 1 -sec retention interval appeared to dissipate as the ITI increased, the transition $\times$ delay $\times$ ITI interaction was not significant $[F(4,28)=1.13]$. Perhaps the failure to observe a significant three-way interaction is related to the fact that as the ITI increased from 5 to $10 \mathrm{sec}$, the amount of divergence between neutral and negative transitions across delays was virtually eliminated in four pigeons, but not in the remaining four. There are data to indicate, however, that the amount of divergence on negative transition trials was significantly reduced as the ITI increased. A single interference score, representing the amount of divergence between neutral and negative transitions across the retention intervals, was calculated for each subject by subtracting negative transition performance from neutral transition performance at each retention interval, and then subtracting the relative amount of interference at the zero delay from the 1-sec delay. A comparison of interference scores at the 5- and 10 -sec ITIs indicated that interference was reduced in magnitude in all subjects as the ITI increased ( $p<.004$, binomial test $)$.

The effect on performance of reversing the position of the correct comparison stimulus from Trial $n-1$ to Trial $n$ was negligible and unsystematic. The position data appear in Table 1. An analysis of variance involving position (same or reversed), delay, and ITI revealed a nonsignificant effect of position $(F<1)$. Furthermore, position did not interact sig- 
Table 1

Mean Percentage Correct on Trial $\mathbf{n}$ when the Correct Comparison Position of Trial $n-1$ was Reversed on Trial $\mathbf{n}$, or the Same on Trial $\mathbf{n}$ as a Function of the ITI, and Sample Duration (D)

\begin{tabular}{|c|c|c|c|c|c|c|}
\hline \multirow{3}{*}{$\begin{array}{c}\text { Sample } \\
\mathrm{D}^{*}\end{array}$} & \multicolumn{6}{|c|}{ ITI Value* } \\
\hline & \multicolumn{2}{|c|}{2} & \multicolumn{2}{|c|}{5} & \multicolumn{2}{|c|}{10} \\
\hline & $S$ & $\mathrm{R}$ & $S$ & $\mathbf{R}$ & $S$ & $\mathbf{R}$ \\
\hline 1.0 & 80.9 & 80.9 & 87.7 & 85.1 & 82.8 & 83.3 \\
\hline .5 & 67.6 & 66.3 & 67.9 & 67.1 & 65.4 & 65.2 \\
\hline
\end{tabular}

Note $-S=$ same position; $R=$ reversed position. $\quad$ *In seconds.

nificantly with delay, ITI, or transition (all Fs $<1$ ).

In general, the results of Phase 1 indicate that the proactive influence of Trial $n-1$ was largely due to reversing the role of the correct and incorrect comparison hue across trials, and not to reversing the positions of the correct and incorrect comparison hues.

\section{Phase 2}

When the sample duration was reduced from 1.0 to $.5 \mathrm{sec}$, overall performance at the 0 -sec retention interval dropped approximately $15 \%$. Consistent with the Phase 1 results, positive transition performance remained comparable to neutral transition performance at each retention interval and at each ITI duration. Also consistent with the Phase 1 results, negative transition performance diverged from neutral transition performance as the retention interval increased from 0 to $1 \mathrm{sec}$, and the divergence was larger at the shorter ITIs.

The data from Phase 2 appear in Figure 2.

An analysis of variance indicated a significant main effect of transition $[F(2,14)=6.87]$ and delay $[F(1,7)=55.2]$ but not $\operatorname{ITI}[F(2,14)=1.34]$. An analysis of the transition effect indicated that the combined performance on positive and neutral transitions was significantly better than performance on

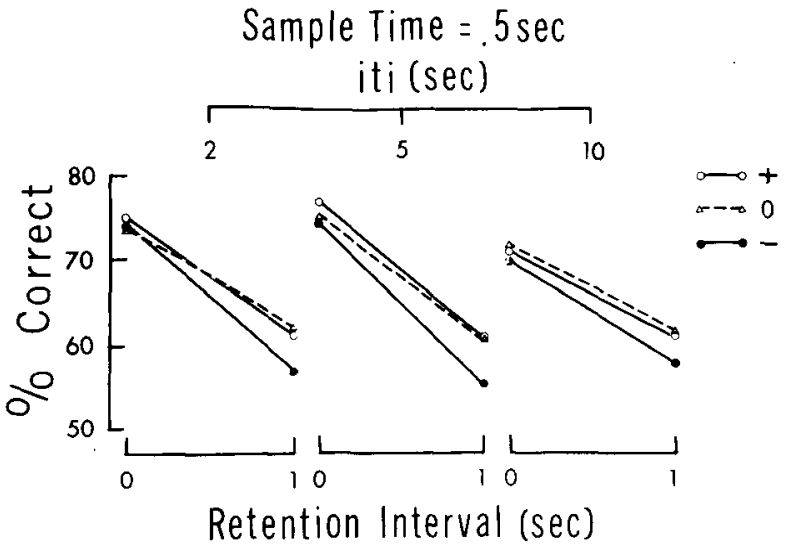

Figure 2. Phase 2: Performance on positive $(t)$, neutral $(0)$, and negative ( - ) transition trials as a function of the retention interval and ITI condition when the sample duration was .5 sec. negative transitions $[F(1,7)=7.13]$. However, performance on positive transitions was not significantly superior to neutral transitions $(\mathrm{F}<1)$.

As in Phase 1, the transition effect interacted with delay $[F(2,14)=6.77]$. More specifically, negative transition performance diverged from the combined performance of positive and neutral transitions as the retention interval increased $[F(1,7)=12.53]$, whereas positive and neutral transition performance did not interact significantly with delay $[F(1,7)=2.57]$. Also consistent with the Phase 1 data was the absence of a significant interaction among transition, delay, and ITI $(F<1)$. However, an inspection of interference scores showed that the amount of interference was reduced in 7 of the 8 pigeons as the ITI increased from 5 to $10 \mathrm{sec}$ ( $p<.035$, binomial test).

Finally, the effect on performance of reversing the position of the correct comparison from Trial $n-1$ to Trial $\mathrm{n}$ had a negligible (nonsignificant) effect on Trial $n$ performance. An analysis of variance involving position, delay, and ITI showed a nonsignificant main effect of position, and position did not interact significantly with transition, delay, or ITI (all Fs $<1$ ) (see Table 1).

\section{DISCUSSION}

The results of the present experiment indicate that a prior delayed matching trial can serve as a source of proactive interference on the subsequent trial, but not as a source of proactive facilitation.

The results add to the growing body of delayed matching studies that have found the prior trial to be a significant source of proactive interference for monkeys (Moise, 1976; Worsham, 1975), dolphins (Herman, 1975), and pigeons (Grant, 1975). The amount of proactive interference that occurs appears to depend on (1) the temporal proximity of the interference trial to the test trial (i.e., in the present study, interference was greater when Trial $n-1$ was closer in time to Trial $\mathrm{n}$, and dissipated as the ITI increased) and (2) the retention interval of Trial $n$ (i.e., the amount of interference, relative to control performance, was greater when the retention interval of Trial $\mathbf{n}$ was relatively long).

The diverging course of forgetting on interference trials (relative to neutral transition trials) appears to be a reliable finding, having been reported when the proactive event was an interfering stimulus presented on the center key before the sample presentation (Grant \& Roberts, 1973; Zentall \& Hogan, 1977) and when the proactive event was the immediately preceding delayed matching trial (Grant, 1975). The present finding, that interference on $1-s e c$ delay trials was reduced as the ITI was increased to $10 \mathrm{sec}$, is also consistent with Grant's (1975) finding. Roberts (1980), on the other hand, used a similar procedure and reported little evidence that interference produced by 
a prior trial was reduced by increasing the ITI. Roberts may have failed to observe decreased interference with increased ITI, however, because he did not analyze his data with delay as a factor. Since interference effects are generally negligible at short delays (e.g., $0 \mathrm{sec}$ ), the interference reduction may have been substantially diluted by pooling over delay.

One must be cautious, of course, in interpreting interactions when performance ceilings might be involved. One could argue that the diverging neutralnegative transition functions found in Phase 1 were an artifact of high performance at the 0 -sec retention interval. If performance is high on negative transition trials, it may not be possible for neutral transition performance to be significantly better. A performance ceiling might also account for the failure to observe facilitation on positive transition trials, relative to neutral transitions, especially at the 0 -sec retention interval (see Figure 1). Examination of the Phase 2 data, however, does not support a ceiling interpretation of either the divergent negative transition performance functions or the absence of proactive facilitation on positive transition trials. As can be seen in Figure 2, when the sample duration was reduced to $.5 \mathrm{sec}$, negative transition performance diverged from neutral transition performance as the retention interval increased, even though 0 -sec delay performance on neutral transition trials was substantially below a performance ceiling. In addition, significant proactive facilitation was absent on positive transition trials during Phase 2 . Thus, the proactive influences of Trial $n-1$ were independent of a measurement ceiling.

In the present study, the effects of trials prior to Trial $\mathbf{n}-\mathbf{1}$ were not considered. One could argue that these trials might have some effect on Trial $\mathrm{n}$ as well. But the present study found that the effects of Trial $\mathrm{n}-1$ on performance on Trial $\mathbf{n}$ were virtually absent following a 10-sec ITI (especially in Phase 1), and, since the end of Trial $n-2$ was separated from the start of Trial $\mathrm{n}$ by from 10 to $25 \mathrm{sec}$, it is unlikely that trials prior to Trial $n-1$ had any effect on Trial $n$ performance.

Both temporal discrimination theory and trace strength theory predict that negative transition performance should diverge from neutral transition performance as the retention interval increases. Also, both theories predict that the amount of divergence should decrease as the ITI increases. The temporal discrimination hypothesis proposed by D'Amato (1973) and supported by Zentall and Hogan (1977) suggests that the decline in delayed matching accuracy with increasing retention interval reflects increased difficulty in discriminating whether the sample stimulus or a competing proactive stimulus had been seen last. The temporal discrimination is relatively easy when the retention interval of Trial $n$ is very brief, but increases in difficulty as the retention interval increases. As the temporal discrimination becomes more difficult, performance decreases.

Trace theory, according to Grant (1975), also predicts that the amount of proactive interference depends on the temporal proximity of the interfering stimulus to the sample stimulus. According to Grant, if the proactive and sample stimuli are turned off at approximately the same time, the respective memory traces should begin to decay at approximately the same time, and an equivalent amount of proactive interference should result at each retention interval. However, if the proactive event is separated in time from the sample, proactive interference should increase with increasing retention interval. This should occur because the difference between the strength of the sample trace and the strength of the proactive stimulus trace would be greater following an immediate retention test (e.g., a 0-sec delay) than following a later retention test (e.g., a 1-sec delay), and, according to trace-decay theory, the more similar two traces are in strength, the greater will be the interference. In addition, Grant suggests that the proactive effect of a prior stimulus should decrease as the time between the proactive stimulus and sample (i.e., the interstimulus interval) increases, since the proactive stimulus trace should decay during that interval. In the present experiment, the proactive stimulus is represented by the prior trial and the interstimulus interval by the ITI.

Improvement in performance associated with increasing the ITI in the present experiment was quite modest. Although performance improved with increased ITI in both phases, the increase was reliable only in Phase 1. In Phase 2, interference produced by negative transitions at the $1-\mathrm{sec}$ retention interval was still present at the 10-sec ITI. Perhaps the limited range of ITI values and short sample duration used in Phase 2 of the present study accounts for the failure of proactive interference to dissipate as the ITI increased. Grant (1975), for example, has shown that proactive interference dissipates almost entirely with ITIs of 20-40 sec.

Although trace strength theory and temporal discrimination theory make similar predictions regarding the detrimental effects of negative transitions, they make different predictions regarding the facilitating effect of positive transitions. Temporal discrimination theory predicts an absence of proactive facilitation on positive transition trials, since a temporal discrimination between a proactive stimulus and the subsequent sample is necessary only when the proactive stimulus appears as a competing alternative during the retention test. On the other hand, trace theory suggests that positive transition trials should facilitate Trial $n$ performance, because the memory trace of Trial $n-1$ should strengthen the 
memory trace of the sample on Trial $n$. According to decay theory (Roberts, 1972), the amount of facilitation should vary inversely with the duration of the ITI. Furthermore, facilitation should be greater after a 0 -sec retention interval on Trial $\mathbf{n}$ than after a $1-\mathrm{sec}$ retention interval, since the reduction in strength of a memory trace is assumed to be a constant proportion of its absolute strength (Roberts; 1972; Roberts \& Grant, 1974). The present data show no evidence for superiority of performance on positive transition trials, relative to neutral transition trials. The absence of proactive facilitation in the present experiment, however, should be interpreted with caution, since it is possible that the amount of facilitation and interference predicted by decay theory are not symmetrical, relative to neutral trials. The simplest mechanism for such asymmetry is a trace strength ceiling. If one assumes that the sample trace on neutral trials is already at asymptote, then the increment in sample trace strength resulting from a positive transition trial may not produce improvement in performance. Negative transitions, however, can produce interference because the sample trace strength is well above its lowest bound. Although such an explanation may account for the findings from Phase 1 , in which performance on neutral transitions was at a high level, it is unlikely that such an explanation can account for the asymmetry in facilitation and interference found in Phase 2, because in Phase 2 performance on neutral transition trials was at a lower level.

Alternatively, trace theory can account for the present findings if one assumes that the proactive effect of Trial $n-1$ on Trial $n$ performance is inversely proportional to the strength of whichever Trial $n$ stimulus is augmented by the prior trial. Thus, because the tendency to respond to the incorrect alternative should, in general, be less than the tendency to respond to the correct alternative, a negative transition trial should increase the strength of the incorrect alternative proportionally more than a positive transition trial should increase the strength of the correct alternative. Further research is needed to test the adequacy of this assumption.

Roberts (1980) has reported that pigeons' delayed matching performance improves as the ITI increases from 1 to $20 \mathrm{sec}$. An examination of two-trial sequences when the ITI was $1 \mathrm{sec}$ revealed that pigeons chose the same comparison stimulus (or position) on Trial $n$ as that chosen on Trial $n-1$. As in the present experiment, performance was generally better when the correct and incorrect comparison stimuli were maintained in the same role on Trial $n-1$ and Trial $n$ (facilitating sequence) than when they were reversed in role across trials (interfering sequence).
However, Roberts also found that performance was generally better when the position of the correct comparison was the same on Trial $n-1$ and Trial $n$ (facilitating sequence) than when the position of the correct comparison was reversed across trials (interfering sequence). In the present experiment, on the other hand, no such position effects were found. Differences in the stimulus dimensions used may account for the differences in results. The sample and comparison stimuli employed by Roberts were line orientations, whereas in the present experiment they were hues. It may be that highly salient colors reduce the extent to which position cues control responding. Future research should examine the effect of stimulus (i.e., sample and comparison) salience on the proactive influence of peck location associated with the last reward.

\section{REFERENCES}

D'Aмлто, M. R. Delayed matching and short-term memory in monkeys. In G. H. Bower (Ed.), The psychology of learning and motivation (Vol. 7). New York: Academic Press, 1973.

Grant, D. S. Proactive interference in pigeon short-term memory. Journal of Experimental Psychology: Animal Behavior Processes, 1975, 104, 207-220.

Grant, D. S., \& Roberts, W. A. Trace interaction in pigeon short-term memory. Journal of Experimental Psychology, 1973, 101, 21-29.

Herman, L. M. Interference and auditory short-term memory in the bottle-nosed dolphin. Animal Learning \& Behavior, 1975, 3, 43-48.

Moise, S. L. Proactive effects of stimuli, delays, and response position during delayed matching from sample. Animal Learning \& Behavior, 1976, 4, 37-40.

Roberts, W. A. Short-term memory in the pigeon: The effects of repetition and spacing. Journal of Experimental Psychology, 1972, 94, 74-83.

RoBe RTS, W. A. Distribution of trials and intertrial retention in delayed matching to sample with pigeons. Journal of Experimental Psychology: Animal Behavior Processes, 1980, 6, 217-237.

Roberts, W. A., \& Grant, D. S. Short-term memory in the pigeon with presentation time precisely controlled. Learning and Motivation, 1974, 5, 393-408.

Roberts, W. A., \& Grant, D. S. Studies of short-term memory in the pigeon using the delayed matching-to-sample procedure. In D. L. Medin, W. A. Roberts, \& R. T. Davis (Eds.), Processes of animal memory. Hillsdale, N.J: Erlbaum, 1976.

Worsham, R. W. Temporal discrimination factors in the delayed matching-to-sample task in monkeys. Animal Learning \& Behavior, 1975, 3, 93-97.

Zentall, T. R., \& Hogan, D. E. Short-term proactive inhibition in the pigeon. Learning and Motivation, 1977, 8, 367-386.

Zentalt, T. R., \& Hogan, D. E. Same/different concept learning in the pigeon: The effect of negative instances and prior adaptation to the transfer stimuli. Journal of the Experimental Analysis of Behavior, 1978, 30, 177-186.

(Manuscript received August 7, 1980; revision accepted for publication May 17, 1981.) 